\title{
CHEMICAL AND BIOLOGICAL INVESTIGATIONS OF THE RED SEA SPONGE NEGOMBATA CORTICATA
}

Safwat Ahmed, Mostafa Mesbah, Diaa Youssef, Sherief Khalifa*

Department of Pharmacognosy, Faculty of Pharmacy, Suez Canal University, Ismailia, Egypt

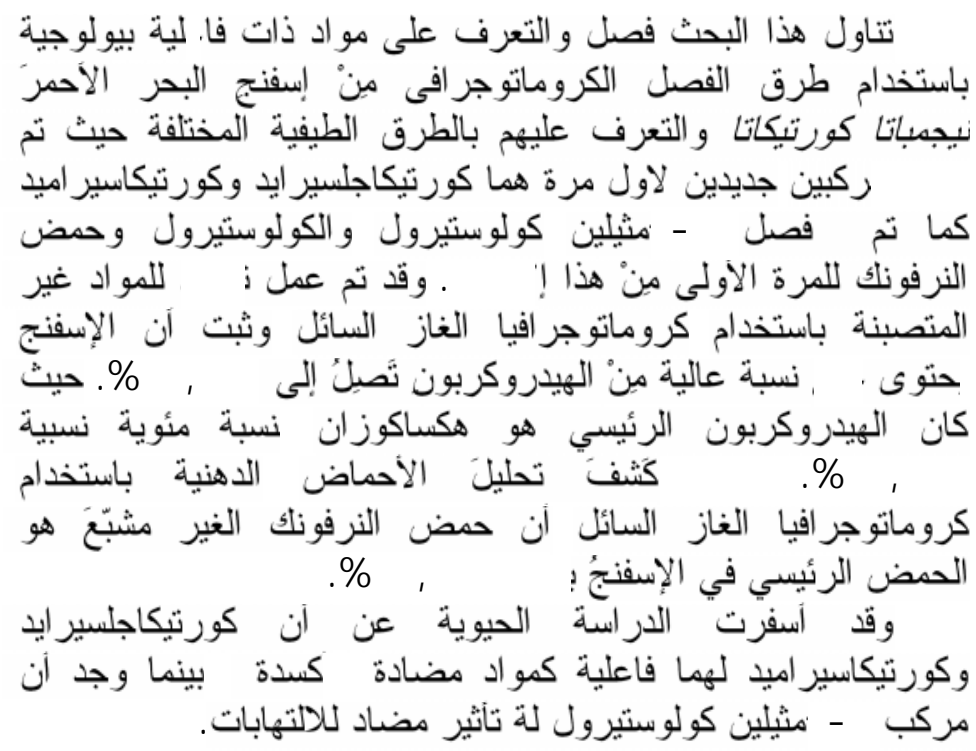

Two new metabolites, a diglyceride ester, corticaglyceride (1) and a sphingolipid, corticaceramide (5) were isolated and characterized from the Red Sea sponge Negombata corticata (Carter). Other previously reported compounds including nervonic acid (2), 24-methylene cholesterol (3) and cholesterol (4) were also isolated for the first time from this genus. GLC analysis of the unsaponifiable matter revealed that it contained a high percentage of hydrocarbons $(89.474 \%)$ with n-hexacosane as a major component (10.979\%). Furthermore, the identified sterol represents 7.162\%. GLC analysis of the fatty acid methyl esters revealed that nervonic acid is the major fatty acid (73.782\%). Compound 3 showed anti-inflammatory activity, while compounds $\mathbf{1}$ and $\mathbf{5}$ showed mild anti-oxidant properties.

Received in 12/3/2006 \& Accepted in 28/5/2006

*Corresponding author. 


\section{INTRODUCTION}

Marine sponges continue to be a rich source of new secondary metabolites with a wide range of biological activities. ${ }^{1}$ Marine natural products chemists have described a wonderful array of pharmacologically-active metabolites from marine sponges. ${ }^{2}$ The genus Negombata is represented in the Red Sea by two species, namely Negombata magnifica (Keller) (formerly Latrunculia magnifica) and Negombata corticata (Carter), family Podospongiidae. ${ }^{3}$ Genus Negombata was shown to be a source of biologically active macrolides. ${ }^{4-8}$

Lipid classes, sterols and fatty acid are potentially excellent biomarker compounds in marine samples due to their stability and diversity of their structures. They are present in all eukaryotes and share with phospholipids a structural function in membranes due to their role in chemotaxonomic purposes and for food web tracing. ${ }^{9}$ Sphingolipids have emerged as a new class of modulators of various cell functions. Ceramides, which are the central molecule in the biosynthesis of sphingolipids and glycosphingolipids, are involved in the regulation of different cellular events, including cell senescence, differentiation, and apoptosis. Many biologically active sphingolipids have been isolated from different marine organisms. ${ }^{10}$

This study describes the investigation of lipoidal content of the sponge Negombata corticata, in addition to the isolation and identification of biologically active compounds. Chemical investigation of the Red Sea Sponge Negombata corticata led to the isolation and structure elucidation of two new metabolites diglyceride ester, corticaglyceride (1) and sphingolipid, corticaceramide (5) along with the previously known metabolites, nervonic acid (2), 24-methylenecholesterol (3) and cholesterol (4) for the first time from genus Negombata. Nervonic acid is the most important of the tetracosanic acids $\left(\mathrm{C}_{24}\right)$ which is a significant component of many sphingolipids. This acid was isolated from Jojoba oil and Honesty seed oil (Lunaria beeiennis). ${ }^{11}$ Nervonic acid is a major component of the human brain. Its level increases in infant brain until the $5^{\text {th }}$ year of age, and may be used as an indicator of brain maturity. Levels of nervonic acid were found to be lower in patients with demyelinating diseases and schizophrenia. Nervonic acid is currently being promoted for regulation of brain cell function and increasing mental activity. ${ }^{12}$

Biological evaluation of isolated metabolites revealed that, $\mathbf{1}$ and $\mathbf{5}$ possess anti-oxidant activity. Compound $\mathbf{3}$ was found to exhibit anti-inflammatory activity.

\section{EXPERIMENTAL}

Biological material, collection and identification

The Negombata corticata, (Carter) (coll. no. SAA-8) was collected by SCUBA at depths of 15 - 
$20 \mathrm{~m}$ from Safaga at the Egyptian Red Sea. The sponge materials were frozen immediately and kept frozen at $-20^{\circ}$ until processed. The sponge material was identified by Prof. Rob. W. M. van Soest, Faculty of Science, Zoological Museum, Amsterdam. A voucher specimen was deposited at the Zoological Museum of the University of Amsterdam, under registration No. ZMAPOR. 18569 and in our Red Sea invertebrates collection at the Department of Pharmacognosy under registration number SAA-8.

\section{General experimental procedures}

The ${ }^{1} \mathrm{H}-$ and ${ }^{13} \mathrm{C}$-NMR spectra were measured on a JEOL 500 spectrometer and mass spectrometry was performed using a QSTAR $^{\circledR}$ hybrid pulsar-i instrument (Applied Biosystems, Foster City, CA) equipped with a nano spray ion source. Stuart Scientific apparatus for melting point determination, Stuart Scientific Co. LTD, Great Britain, USA. The UV spectra were measured by a double - beam Shimadzu UVVisible spectrophotometer (model UV- 1601 PC, Japan). IR spectra were measured by Nicolit FT IR spectrophotometer range 4004000,USA.

Fatty acid methyl esters were identified using Hewlett Packard (HP) gas liquid chromatography, series 6890 equipped with Flame Ionization Detector (FID). A capillary column (HP-INNOWAX, Polyethylene Glycol, 30 m x $530 \mu \mathrm{m}$, film thickness $1.00 \mu \mathrm{m}$ ) was used in separation of fatty acids. The injector port temperature was set at $250^{\circ}$ (splitless mode) and a pressure of $14.81 \mathrm{psi}$ and the detector cell at $275^{\circ}$. The flow rate of the carrier gas $\left(\mathrm{N}_{2}\right)$ was $30 \mathrm{~mL} / \mathrm{min}$. The initial column temperature was $70^{\circ}$ and increased to $200^{\circ}$ by the rate of $4 \% \mathrm{~min}$., then isothermally for a total run time of $32.5 \mathrm{~min}$.

Unsaponifiable matters were identified using capillary column (HP-1 methyl siloxane, 30m x 350 $\mu \mathrm{m}$, film thickness $2.65 \mu \mathrm{m}$ ) was used for separation. The injection port temperature was set at $260^{\circ}$ (splitless mode) and the detector cell at $300^{\circ}$. The flow rate of the carrier gas $\left(\mathrm{N}_{2}\right)$ was $6 \mathrm{~mL} / \mathrm{min}$. The initial column temperature was $70^{\circ}$ for $2 \mathrm{~min}$ and increased to $280^{\circ}$ at a rate $10^{\circ} / \mathrm{min}$, then isothermally for a total run time of $23 \mathrm{~min}$.

\section{Chromatographic materials}

Pre-coated silica gel G-25 UV 254 plates were used for thin layer chromatography $(20 \times 20 \mathrm{~cm})$ (E. Merck). Silica gel 60/230-400 mesh was used for column chromatography (EM Science). Gel permeation chromatography was carried out using Sephadex LH-20.

\section{Experimental animals}

Male albino rats weighing between $125-150 \mathrm{~g}$ were used.

\section{Extraction and Isolation}

The voucher specimen of the sponge Negombata corticata was freeze dried (400 gm dry weight), grounded and extracted with a 
mixture of $\mathrm{MeOH} / \mathrm{CH}_{2} \mathrm{Cl}_{2}$ (1:1) $(3 \times 2$ L) at room temperature. The extract was evaporated under vacuum to afford $100 \mathrm{~g}$ of red oil. This extract was subjected to vacuum liquid chromatography on a flash silica gel using hexane, ethyl acetate and methanol gradient.

The fraction eluted using $10 \%$ ethyl acetate in hexane was concentrated to afford $5 \mathrm{~g}$ of reddish residue. Purification of this fraction was carried out by flash column chromatography on silica gel using hexane/ethyl acetate (95:5). Fractions with the same TLC pattern were combined and rechromatographed by flash column chromatography on silica gel using hexane/ethyl acetate (95:5). Final purification was carried out on Sephadex LH-20 using $\mathrm{MeOH} / \mathrm{CHCl}_{3}$ (1:1) to afford 1 (250 $\mathrm{mg})\left(\mathrm{R}_{\mathrm{f}}=0.72,10 \%\right.$ ethyl acetate / hexane).

The fraction eluted with $20 \%$ ethyl acetate in hexane was concentrated to afford $7 \mathrm{~g}$ of reddish residue. Purification of this fraction was carried out by flash column chromatography on silica gel using hexane/ethyl acetate (9:1), resulting in two fractions. The less polar fraction was rechromatographed by flash column chromatography on silica gel using hexane/ethyl acetate (9:1). Final purification was carried out on Sephadex LH-20 using $\mathrm{MeOH} / \mathrm{CHCl}_{3}$ (1:1) to afford 2 (100 $\mathrm{mg})\left(\mathrm{R}_{\mathrm{f}}=0.65,20 \%\right.$ ethyl acetate / hexane).

The more polar fraction was rechromatographed by flash column chromatography on silica gel using hexane/ethyl acetate (9:1). Final purification was carried out on Sephadex $\mathrm{LH}-20$ using $\mathrm{MeOH} / \mathrm{CHCl}_{3}$ $(1: 1)$ to afford $3(80 \mathrm{mg})\left(\mathrm{R}_{\mathrm{f}}=0.5\right.$, $20 \%$ ethyl acetate / hexane) and 4 (4 g) $\left(\mathrm{R}_{\mathrm{f}}=0.5,20 \%\right.$ ethyl acetate / hexane)

The fractions eluted with $30-60 \%$ ethyl acetate in hexane were combined and concentrated to afford $10 \mathrm{~g}$ of reddish residue. Purification of this fraction was carried out by flash column chromatography on silica gel using hexane/ethyl acetate $(85: 15)$. Fractions with the same TLC pattern were combined and rechromatographed by flash column chromatography on silica gel using hexane/ethyl acetate (85:15). This fraction was subjected to flash column chromatography on silica gel using 2\% methanol in chloroform. Final purification was carried out on Sephadex $\mathrm{LH}-20$ using $\mathrm{MeOH} / \mathrm{CHCl}_{3}$ (1:1) to afford 5 (45 mg) $\left(\mathrm{R}_{\mathrm{f}}=0.66\right.$, $10 \%$ methanol in chloroform).

\section{Investigation of lipoidal matter}

The extracted lipoidal matter (2 gm hexane fraction) of the sponge was saponified..$^{13}$ The unsaponifiable fraction was separated and weighed $(1.2 \mathrm{~g})$. A two-percentage chloroformic solution of the unsaponifiable matters was analyzed by GLC technique. Identification of the hydrocarbons and sterols was carried out by comparing the relative retention time of the peaks with those of the pure available authentics. The quantitative estimation of each peak 
was done by peak area measurement using a computing integrator. Results are shown in Table 1.

The aqueous alkaline solution (aqueous mother liquor and washings) left after the separation of the unsaponifiable matters was acidified with concentrated hydrochloric acid (litmus paper). The liberated acids $(0.4 \mathrm{~g})$ were extracted with $(4 \times 50 \mathrm{ml})$ ether and their methyl esters were prepared. ${ }^{14}$ A twopercentage chloroformic solution of the prepared fatty acid methyl ester (FAME) was analyzed by GLC. Identification of the fatty acids was carried out by comparing the relative retention time of the peaks with those of the pure available authentic standards. The quantitative estimation of each peak was done by peak area measurement using a computing integrator. Results are shown in Table 2.

\section{Hydrolysis of 1 and 5}

Compound 1 was refluxed with $10 \%$ ethanolic $\mathrm{KOH}$ and benzene for $24 \mathrm{~h}$. After adding $\mathrm{H}_{2} \mathrm{O}$, the reaction mixture was extracted with ether. The aqueous alkaline solution was acidified with concentrated $\mathrm{HCl}$. The reaction mixture was extracted with ether. The resulting ether-soluble fraction was concentrated to yield a fatty acid. Compound 5 was cleaved by the same method.

\section{Preparation of fatty acid methyl esters of 1, 2 and 5}

Prepared fatty acid of $\mathbf{1}$ was refluxed with $\mathrm{MeOH}$ and concentrated $\mathrm{H}_{2} \mathrm{SO}_{4}$ for $1 \mathrm{~h}$. After adding $\mathrm{H}_{2} \mathrm{O}$, the reaction mixture was extracted with ether and dried with anhydrous magnesium sulfate. The resulting ether-soluble fraction was concentrated to yield a fatty acid methyl ester, which was analyzed by GlC. Fatty acid methyl esters of 2 and 5 were prepared by the same method.

Compound (1): colorless oil; $\mathrm{R}_{\mathrm{f}}=$ $0.72,10 \%$ ethyl acetate / hexane; UV $(\mathrm{MeOH}) \lambda_{\max }(\log \epsilon) 222 \mathrm{~nm}$; IR $(\mathrm{KBr})$ (thin film) $v_{\max } 3503.94$, 2127.47, 1654.92, 1437.50, 1030.83, $953.39 \mathrm{~cm}^{-1}$; MS: $\mathrm{m} / z 703[\mathrm{M}-\mathrm{H}]^{+}$ $\left(\mathrm{C}_{45} \mathrm{H}_{84} \mathrm{O}_{5}\right) ;{ }^{1} \mathrm{H}-\mathrm{NMR}\left(\mathrm{CDCl}_{3}, 500\right.$ $\mathrm{MHz})$ and ${ }^{13} \mathrm{C}-\mathrm{NMR}\left(\mathrm{CDCl}_{3}, 125\right.$ $\mathrm{MHz}$ ), see Table 3.

Compound (2): White solid; $\mathrm{R}_{\mathrm{F}}=$ $0.65,20 \%$ ethyl acetate / hexane; m.p 43; UV (MeOH) $\lambda_{\max }(\log \epsilon) 218 \mathrm{~nm}$; IR (KBr) (thin film) $v_{\text {max }} 2918.11$, 2848.97, 1704.56, 1466.61, 1296.55, 1433.50, $723.44 \quad \mathrm{~cm}^{-1}$; MS: $\mathrm{m} / 2$ $349[\mathrm{M}-\mathrm{OH}]^{+} \quad\left(\mathrm{C}_{24} \mathrm{H}_{46} \mathrm{O}_{2}\right) ;{ }^{1} \mathrm{H}-\mathrm{NMR}$ $\left(\mathrm{CDCl}_{3}, 500 \mathrm{MHz}\right)$ and ${ }^{13} \mathrm{C}-\mathrm{NMR}$ $\left(\mathrm{CDCl}_{3}, 125 \mathrm{MHz}\right)$, see Table 4 .

Compound (3): White solid; $\mathrm{R}_{\mathrm{f}}=0.5$, $20 \%$ ethyl acetate / hexane; m.p130 $\mathrm{UV}(\mathrm{MeOH}) \lambda_{\max }\left(\log _{\epsilon}\right) 220 \mathrm{~nm}$; IR $(\mathrm{KBr}) \quad\left(\right.$ thin film) $v_{\mathrm{max}} 3426.34$, 2935.23, 1708,69, 1462.80, 1050.06 $\mathrm{cm}^{-1}$; MS: $\mathrm{m} / z 397[\mathrm{M}-\mathrm{H}]^{+}\left(\mathrm{C}_{28} \mathrm{H}_{48} \mathrm{O}\right)$.

Compound (4): White solid; $\mathrm{R}_{\mathrm{f}}=0.5$, $20 \%$ ethyl acetate / hexane; m.p133; $\mathrm{UV}(\mathrm{MeOH}) \lambda_{\max }(\log \epsilon) 220 \mathrm{~nm}$; IR $(\mathrm{KBr}) \quad$ (thin film) $v_{\max } \quad 3444.34$ 2856.86, 1648.42, 1461.80, 1376.18, 
$1130.10 \mathrm{~cm}^{-1}$; MS: $\mathrm{m} / z 399[\mathrm{M}+\mathrm{H}]^{+}$ $\left(\mathrm{C}_{27} \mathrm{H}_{48} \mathrm{O}\right)$.

Compound (5): White solid; $\mathrm{R}_{\mathrm{f}}=$ $0.66,10 \%$ methanol in chloroform; m.p73; UV (MeOH) $\lambda_{\max }(\log \epsilon) 235$ $\mathrm{nm}$; IR (KBr) (thin film) $v_{\max } 3410.93$, 3332.37, 2920.67, 2848.51, 1642.24, $1404.98,1130.75,1049.30,725.06$ $\mathrm{cm}^{-1}$; MS: $\mathrm{m} / \mathrm{z} 536.4807 \quad[\mathrm{M}-\mathrm{H}]^{+}$ $\left(\mathrm{C}_{34} \mathrm{H}_{65} \mathrm{O}_{3} \mathrm{~N}\right) ;{ }^{1} \mathrm{H}-\mathrm{NMR}\left(\mathrm{CDCl}_{3}, 500\right.$ $\mathrm{MHz})$ and ${ }^{13} \mathrm{C}-\mathrm{NMR} \quad\left(\mathrm{CDCl}_{3}, 125\right.$ $\mathrm{MHz}$ ), see Table 5.

\section{Biological evaluation}

1- Anti-inflammatory test for compound 3

Hind paw-oedema method was used where $4 \%$ formalin solution was used to induce paw skin oedema. A group of animals (5 rats) were given dexamethasone subcutaneously at a dose of $20 \mathrm{mg} / \mathrm{kg}$. A second group (5 rats) were injected test compound $\mathbf{3}$ subcutaneously between the sholder blades with a dose of $20 \mathrm{mg} / \mathrm{kg}$. Five rats were taken as control group. ${ }^{15}$

Percentage increase in paw thickness were calculated and presented as mean \pm SE (Table 6). The data were computed using SPSS program and analyzed by one-way analysis of variance (ANOVA) followed by PostHoc test (Bonfrroni) for multiple comparisons. Significant results were determined as $\mathrm{p}<0.05$.

\section{2- Anti-oxidant test}

TLC-based 1,1-diphenyl-2-picrylhydrazyl radical (DPPH) autographic chemical assay was used. Isolated compounds were dissolved in DMF at a concentration of $2 \mathrm{mg} / \mathrm{mL}$. A $4 \mu \mathrm{l}$ volume of each compound was applied in the form of a spot (4-5 mm in diameter) on silica gel GF plates. The residual DMF was removed under vacuum (15-20 min). A similar amount of Vitamin E in DMF was used as positive antioxidant control. The radical-scavenging effects of the isolated compounds were detected on the TLC plate using a spray reagent composed of a $0.2 \%(\mathrm{w} / \mathrm{v})$ solution of 1,1-diphenyl-2-picrylhydrazyl radical $(\mathrm{DPPH})$ in $\mathrm{MeOH}$. The plate was observed $30 \mathrm{~min}$ after spraying. Active compounds were observed as yellow spots against a purple background. Relative radicalscavenging activity was assigned as "strong" (compounds that produce an intense bright yellow zone), "medium" (compounds that produce a clear yellow spot), "weak" (compounds that produce a weakly visible yellow spot), or "not active" (compounds that produce no sign of any yellow spot). ${ }^{16}$ Vitamin E was taken as positive antioxidant control which produced an intense bright yellow zone. 
Table 1: GLC analysis of the unsaponifiable matter of the Red Sea sponge Negombata corticata.

\begin{tabular}{|l|c|c|}
\hline \multicolumn{1}{|c|}{ Identified compounds $\left(\mathrm{C}_{\mathrm{n}}\right)$} & $\mathrm{RR}_{\mathrm{t}}{ }^{*}$ & Relative\% \\
\hline n-Tridecane $\left(\mathrm{C}_{13}\right)$ & 0.358 & 2.938 \\
\hline n-Tetradecane $\left(\mathrm{C}_{14}\right)$ & 0.407 & 2.874 \\
\hline n- Pentadecane $\left(\mathrm{C}_{15}\right)$ & 0.485 & 2.880 \\
\hline n- Hexadecane $\left(\mathrm{C}_{16}\right)$ & 0.528 & 3.0 \\
\hline n-Heptadecane $\left(\mathrm{C}_{17}\right)$ & 0.589 & 3.12 \\
\hline n-Octadecane $\left(\mathrm{C}_{18}\right)$ & 0.640 & 3.654 \\
\hline n-Nonadecane $\left(\mathrm{C}_{19}\right)$ & 0.692 & 3.638 \\
\hline n-Eicosane $\left(\mathrm{C}_{20}\right)$ & 0.743 & 2.99 \\
\hline n-Heneicosane $\left(\mathrm{C}_{21}\right)$ & 0.769 & 4.241 \\
\hline n-Docosane $\left(\mathrm{C}_{22}\right)$ & 0.785 & 3.604 \\
\hline n-Tricosane $\left(\mathrm{C}_{23}\right)$ & 0.829 & 5.828 \\
\hline n-Tetracosane $\left(\mathrm{C}_{24}\right)$ & 0.837 & 6.594 \\
\hline n-Pentacosane $\left(\mathrm{C}_{25}\right)$ & 0.903 & 7.739 \\
\hline n-Hexacosane $\left(\mathrm{C}_{26}\right)$ & 1 & 10.979 \\
\hline n-Heptacosane $\left(\mathrm{C}_{27}\right)$ & 1.776 & 5.058 \\
\hline n-Octacosane $\left(\mathrm{C}_{28}\right)$ & 1.093 & 3.566 \\
\hline n-Nonacosane $\left(\mathrm{C}_{29}\right)$ & 1.143 & 3.947 \\
\hline n-Tricontane $\left(\mathrm{C}_{30}\right)$ & 1.777 & 5.757 \\
\hline n- Hentricontane $\left(\mathrm{C}_{31}\right)$ & 1.221 & 3.49 \\
\hline n-Docontane $\left(\mathrm{C}_{32}\right)$ & 1.256 & 3.578 \\
\hline Cholesterol $\left(\mathrm{C}_{27}\right)$ & 1.329 & 1.759 \\
\hline Campsterol $\left(\mathrm{C}_{28}\right)$ & 1.402 & 2.622 \\
\hline Stigmasterol $\left(\mathrm{C}_{29}\right)$ & 1.489 & 1.4322 \\
\hline$\beta$-Sitosterol $\left(\mathrm{C}_{29}\right)$ & 1.507 & 1.349 \\
\hline B-Amyrin $\left(\mathrm{C}_{30}\right)$ & 1.561 & 3.369 \\
\hline Percentage of total hydrocarbons & & 89.474 \\
\hline Percentage of total sterols & & 7.162 \\
\hline & & \\
\hline
\end{tabular}

$\mathrm{RR}_{\mathrm{t}}$ *: Relative retention time $\mathrm{n}$-hexacosane with $\mathrm{R}_{\mathrm{t}}=1 \mathrm{~min}$. 
Table 2: Results of GLC analysis of FAME of the Red Sea sponge Negombata corticata:

\begin{tabular}{||l|c|c|}
\hline \multicolumn{1}{|c|}{$\begin{array}{c}\text { Authentic of FAME } \\
\text { ( } \text { n., no. of unsaturation) }\end{array}$} & RRt* & Relative\% \\
\hline Myrestic acid (C14, 0) & 0.551 & 1.380 \\
\hline Tetradecenoic acid $(\mathrm{C} 14,1)$ & 0.533 & 1.2379 \\
\hline Palmitic acid $(\mathrm{C} 16,0)$ & 0.606 & 1.800 \\
\hline Oleic acid $(\mathrm{C} 18,1)$ & 0.674 & 11.7331 \\
\hline Linolenic acid $(\mathrm{C} 18,3)$ & 0.650 & 1.306 \\
\hline Arachidic acid $(\mathrm{C} 20,0)$ & 0.682 & 4.751 \\
\hline Erucic $(\mathrm{C} 22: 1)$ & 0.757 & 3.021 \\
\hline Behenic acid $(\mathrm{C} 22,0)$ & 0.767 & 1.048 \\
\hline Nervonic acid $(\mathrm{C} 24,1)$ & 1 & 73.782 \\
\hline Percentage of total saturated FAME & & 8.97 \\
\hline Percentage of total unsaturated FAME & & 91.03 \\
\hline \hline
\end{tabular}

$\mathrm{RR}_{\mathrm{t}}{ }^{*}$ : Relative retention time to nervonic acid with $\mathrm{R}_{\mathrm{t}}=1 \mathrm{~min}$.

FAME: Fatty acid methyl esters.

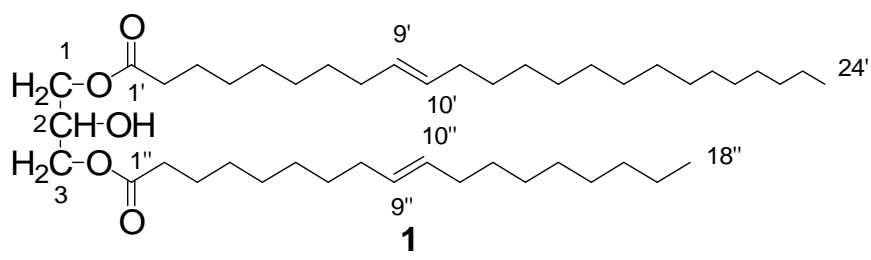<smiles>CCCCCCCCCCCCCCCCCCCCCCCCCCCCCC(=O)O</smiles><smiles>C=C(C)CCC1CCC2C3CC=C4CC(O)CCC4(C)C3CCC12C</smiles>

3<smiles>CC(C)CCCC1CCC2C3CC=C4CC(O)CCC4(C)C3CCC12C</smiles>

O

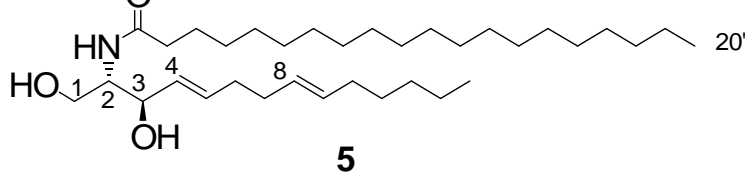


Table 3: ${ }^{13} \mathrm{C}(125 \mathrm{MHz}),{ }^{1} \mathrm{H}(500 \mathrm{MHz})$, COSY and HMBC NMR spectral data of compound 1 in $\mathrm{CDCl}_{3}$.

\begin{tabular}{|c|c|c|c|c|}
\hline No. & $\delta_{\mathrm{C}}$ & $\delta_{\mathrm{H}}$ & $\operatorname{COSY}(\mathrm{H}-\mathrm{H})$ & $\mathrm{HMBC}(\mathrm{H}-\mathrm{C})$ \\
\hline 1 & $62.2^{\mathrm{a}}$ & $\begin{array}{l}4.27(\mathrm{dd}) \\
4.29(\mathrm{dd})\end{array}$ & 2 & 2 \\
\hline 2 & 68.9 & $5.20(q)$ & 1,3 & \\
\hline 3 & $62.2^{a}$ & $\begin{array}{l}4.23(\mathrm{dd}) \\
4.24(\mathrm{dd})\end{array}$ & 2 & 2 \\
\hline $1^{\prime}$ & 173.4 & & & \\
\hline $2^{\prime}$ & 34.3 & $2.31(\mathrm{~m})$ & $3^{\prime}$ & $1^{\prime}, 3^{\prime}, 4^{\prime}$ \\
\hline $3^{\prime}$ & 24.9 & $1.59(\mathrm{~m})$ & $2^{\prime}, 4^{\prime}$ & $1^{\prime}, 2^{\prime}, 4^{\prime}, 5^{\prime}$ \\
\hline $4^{\prime}-7^{\prime}$ & $29.6^{b}$ & $1.24(\mathrm{~m})$ & & \\
\hline $8^{\prime}$ & $27.3^{\mathrm{c}}$ & $1.99(\mathrm{~m})$ & $7^{\prime}, 9^{\prime}$ & $6^{\prime}, 7^{\prime}, 9^{\prime}, 10^{\prime}$ \\
\hline $9^{\prime}$ & 130.9 & $5.33(\mathrm{dt})$ & $8^{\prime}, 10^{\prime}$ & $7^{\prime}, 8^{\prime}, 10^{\prime}, 11^{\prime}$ \\
\hline $10^{\prime}$ & 128.8 & $5.33(\mathrm{dt})$ & $9^{\prime}, 11^{\prime}$ & $8^{\prime}, 9^{\prime}, 11^{\prime}, 12^{\prime}$ \\
\hline $11^{\prime}$ & $27.3^{c}$ & $1.99(\mathrm{~m})$ & $10^{\prime}, 12^{\prime}$ & $9^{\prime}, 10^{\prime}, 12^{\prime}, 13^{\prime}$ \\
\hline $12^{\prime}-21^{\prime}$ & $29.6^{b}$ & $1.24(\mathrm{~m})$ & & \\
\hline $22^{\prime}$ & 32.9 & $1.24(\mathrm{~m})$ & $21^{\prime}, 23^{\prime}$ & $20^{\prime}, 21^{\prime}, 23^{\prime}, 24^{\prime}$ \\
\hline $23^{\prime}$ & 22.7 & $1.24(\mathrm{~m})$ & $22^{\prime}, 24^{\prime}$ & $21^{\prime}, 22^{\prime}, 24^{\prime}$ \\
\hline $24^{\prime}$ & 14.2 & $0.87(\mathrm{t})$ & $23^{\prime}$ & $23^{\prime}$ \\
\hline $1^{\prime \prime}$ & 173.0 & & & \\
\hline $2^{\prime \prime}$ & 34.3 & $2.31(\mathrm{~m})$ & 3" & 1", 3", 4" \\
\hline $3^{\prime \prime}$ & 24.9 & $1.59(\mathrm{~m})$ & 2", 4" & 1", 2", 4", 5" \\
\hline $4^{\prime \prime}-7^{\prime \prime}$ & $29.6^{\mathrm{b}}$ & $1.24(\mathrm{~m})$ & & \\
\hline $8^{\prime \prime}$ & $27.3^{c}$ & $1.99(\mathrm{~m})$ & 7", 9" & 6", 7", 9", 10" \\
\hline $9^{\prime \prime}$ & 129.9 & $5.33(\mathrm{dt})$ & 8", 10" & 7", 8", 10", 11" \\
\hline $10^{\prime \prime}$ & 128.0 & $5.33(\mathrm{dt})$ & 9", 11" & 8", 9", 11", 12" \\
\hline $11^{\prime \prime}$ & $27.3^{c}$ & $1.99(\mathrm{~m})$ & $10 ", 12 "$ & 9", 10", 12",13" \\
\hline $12^{\prime \prime}-15^{\prime \prime}$ & $29.6^{\mathrm{b}}$ & $1.24(\mathrm{~m})$ & & \\
\hline $16^{\prime \prime}$ & 32.9 & $1.24(\mathrm{~m})$ & 15", 17" & 14", 15", 17", 18" \\
\hline $17^{\prime \prime}$ & 22.7 & $1.24(\mathrm{~m})$ & $16 ", 18 "$ & $15^{\prime \prime}, 16^{\prime \prime}, 18^{\prime \prime}$ \\
\hline $18^{\prime \prime}$ & 14.2 & $0.87(\mathrm{t})$ & $17 "$ & $17^{\prime \prime}$ \\
\hline
\end{tabular}

${ }^{\text {a, b }, ~ c}$ Signals with same $\delta$ value in each column are overlapped signals. 
Table 4: ${ }^{13} \mathrm{C}(125 \mathrm{MHz}),{ }^{1} \mathrm{H}(500 \mathrm{MHz})$ and COSY NMR spectral data of compound 2 in $\mathrm{CDCl}_{3}$.

\begin{tabular}{||l|c|c|l||}
\hline \multicolumn{1}{|c|}{ No. } & $\delta_{\mathrm{C}}$ & $\delta_{\mathrm{H}}$ & \multicolumn{1}{c||}{$\operatorname{COSY}(\mathrm{H}-\mathrm{H})$} \\
\hline 1 & 180.2 & & \\
\hline 2 & 34.2 & $2.31(\mathrm{~m})$ & 3 \\
\hline 3 & 24.8 & $1.59(\mathrm{~m})$ & 2,4 \\
\hline $4-7$ & $29.6^{\mathrm{a}}$ & $1.24(\mathrm{~m})$ & \\
\hline 8 & $27.3^{\mathrm{b}}$ & $1.99(\mathrm{~m})$ & 7,9 \\
\hline 9 & 130.9 & $5.33(\mathrm{dt})$ & 8,10 \\
\hline 10 & 128.8 & $5.33(\mathrm{dt})$ & 9,11 \\
\hline 11 & $27.3^{\mathrm{b}}$ & $1.99(\mathrm{~m})$ & 10,12 \\
\hline $12-21$ & $29.6^{\mathrm{a}}$ & $1.24(\mathrm{~m})$ & \\
\hline 22 & 32.0 & $1.24(\mathrm{~m})$ & 21,23 \\
\hline 23 & 22.8 & $1.24(\mathrm{~m})$ & 22,24 \\
\hline 24 & 14.2 & $0.87(\mathrm{t})$ & 23 \\
\hline
\end{tabular}

${ }^{\mathrm{a}, \mathrm{b}}$ Signals with same $\delta$ value are overlapped signals.

Table 5: ${ }^{13} \mathrm{C}(125 \mathrm{MHz}),{ }^{1} \mathrm{H}(500 \mathrm{MHz}), \mathrm{COSY}$ and HMBC NMR spectral data of compound 5 in $\mathrm{CDCl}_{3}$.

\begin{tabular}{|c|c|c|c|c|}
\hline No. & $\delta_{\mathrm{C}}$ & $\delta_{\mathrm{H}}\left(\right.$ mult., $\left.J_{\mathrm{Hz}}\right)$ & COSY $(\mathrm{H}-\mathrm{H})$ & HMBC (H-C) \\
\hline 1 & 62.5 & $3.95(\mathrm{~d}, 11.40), 3.70(\mathrm{br} \mathrm{d})$ & 2 & \\
\hline 2 & 54.0 & $3.91(\mathrm{dt}, 7.65,3.85)$ & 1,3 & \\
\hline 3 & 76.8 & $4.30(\mathrm{br} \mathrm{m})$ & 2,4 & 4,5 \\
\hline 4 & 129.2 & $5.50(\mathrm{dd}, 15.00,6.10)$ & 3,5 & $3,5,6$ \\
\hline 5 & 129.0 & $5.79(\mathrm{dt}, 15.00,7.00)$ & 4,6 & $3,4,6$ \\
\hline 6 & 32.4 & $2.11(\mathrm{dt}, 21.10,6.50)$ & 5,7 & $4,5,7,8$ \\
\hline 7 & 32.2 & $2.08(\mathrm{dt}, 21.10,6.50)$ & 6,8 & $5,6,8,9$ \\
\hline 8 & 133.6 & $5.40^{\mathrm{c}}(\mathrm{dt}, 15.30,10.00)$ & 7,9 & $6,7,9,10$ \\
\hline 9 & 131.4 & $5.40^{\mathrm{c}}(\mathrm{dt}, 15.30,10.00)$ & 8,10 & $7,8,10,11$ \\
\hline 10 & 32.6 & $1.95(\mathrm{dd}, 10.30,6.80)$ & 9,11 & $8,9,11,12$ \\
\hline 11 & 29.6 & $1.24^{\mathrm{a}}(\mathrm{m})$ & 10,12 & $9,10,12,13$ \\
\hline 12 & 32.0 & $1.24^{\mathrm{a}}(\mathrm{m})$ & 11,13 & $10,11,13,14$ \\
\hline 13 & 22.7 & $1.24^{\mathrm{a}}(\mathrm{m})$ & 12,14 & $11,12,14$ \\
\hline 14 & 14.2 & $0.88^{\mathrm{b}}(\mathrm{m})$ & 13 & 12,13 \\
\hline $1^{\prime}$ & 174.0 & & & \\
\hline $2^{\prime}$ & 36.9 & $2.20(\mathrm{t}, 7.60), 2.10$ & $3^{\prime}$ & $1^{\prime}, 3^{\prime}, 4^{\prime}$ \\
\hline $3^{\prime}$ & 25.8 & $1.63(\mathrm{~m})$ & $2,4^{\prime}$ & $2^{\prime}, 44^{\prime}, 5^{\prime}$ \\
\hline 4'-17' & 29.6 & $1.24^{\mathrm{a}}(\mathrm{m})$ & & \\
\hline $18^{\prime}$ & 32.0 & $1.24^{\mathrm{a}}(\mathrm{m})$ & $17^{\prime}, 19^{\prime}$ & $16^{\prime}, 17^{\prime}, 19^{\prime}, 20^{\prime}$ \\
\hline $19^{\prime}$ & 22.7 & $1.24^{\mathrm{a}}(\mathrm{m})$ & $18^{\prime}, 20^{\prime}$ & $17^{\prime}, 18^{\prime}, 20^{\prime}$ \\
\hline $20^{\prime}$ & 14.2 & $0.88^{\mathrm{b}}(\mathrm{m})$ & $19^{\prime}$ & $18^{\prime}, 19^{\prime}$ \\
\hline $\mathrm{OH}$ & & 2.74 (br s) & & \\
\hline $\mathrm{OH}$ & & $2.78(\mathrm{br} \mathrm{s})$ & & \\
\hline $\mathrm{NH}$ & & $6.25($ br d, 7.6$)$ & & $1^{\prime}$ \\
\hline
\end{tabular}

${ }_{\mathrm{a}, \mathrm{b}, \mathrm{c}}$ Signals with same $\delta$ value are overlapped signals. 
Table 6: Comparison of the anti- inflammatory activity of compound 3 against dexamethasone.

\begin{tabular}{|c|c|c|}
\hline Compound 3 & Dexamethasone control & Inflammation control \\
\hline $33.12 \pm 5.35^{*}$ & $35.17 \pm 4.55^{*}$ & $86.73 . \pm 11.09$ \\
\hline
\end{tabular}

$* \mathrm{P}<0.05$ significant difference from Inflammation control

\section{RESULTS AND DISCUSSION}

Compound 1: The structure elucidation of $\mathbf{1}$ began with an analysis of its ${ }^{13} \mathrm{C}$-NMR spectrum and DEPT which indicated the presence of two oxygenated methylenes at $\delta$ 62.2 and $\delta 62.2$, one oxgynated methene at $\delta 68.9$ together with two $\mathrm{CO}$ for two ester moieties at $\delta 173.4$ and 173.0. Also the presence of two disubstituted olefinic moieties were confirmed by ${ }^{13} \mathrm{C}$-NMR resonances at $\delta 130.9,129.9,128.8$ and 128.0. The above mentioned findings were consistent with the presence of a diglyceride ester. GC analysis of the fatty acid methyl ester of compound $\mathbf{1}$ was carried out after hydrolysis. Identification of the fatty acids was carried out by GLC analysis of fatty acid methyl esters by comparing the relative retention time of the peaks with those of the pure available authentic samples. GLC analysis revealed that 1 contains the unsaturated fatty acids, nervonic acid and oleic acid. The placement of the fatty acid at C-1 and C-3 of the diglyceride ester was assigned by ${ }^{1} \mathrm{H}$ ${ }^{1} \mathrm{H}$ COSY between $\mathrm{H}-2$ and $\mathrm{H}_{2}-1$ and $\mathrm{H}-2$ and $\mathrm{H}_{2}-3$. This was confirmed by HMBC of $\mathrm{H}_{2}-1 / \mathrm{C}-2\left({ }^{2} J_{\mathrm{CH}}\right), \mathrm{H}_{2}-1 / \mathrm{C}-3$ $\left({ }^{3} J_{\mathrm{CH}}\right), \quad \mathrm{H}_{2}-1 / \mathrm{C}-1^{\prime} \quad\left({ }^{3} J_{\mathrm{CH}}\right), \quad \mathrm{H}_{2}-3 / \mathrm{C}-2$ $\left({ }^{2} J_{\mathrm{CH}}\right), \mathrm{H}_{2}-3 / \mathrm{C}-1 \quad\left({ }^{3} J_{\mathrm{CH}}\right), \mathrm{H}_{2}-3 / \mathrm{C}-1 "$ $\left({ }^{3} J_{\mathrm{CH}}\right), \mathrm{H}-2 / \mathrm{C}-1\left({ }^{2} J_{\mathrm{CH}}\right), \mathrm{H}-2 / \mathrm{C}-3\left({ }^{2} J_{\mathrm{CH}}\right)$ revealed that $\mathbf{1}$ is 1,3 diglyceride ester (Fig. 1). Detailed analysis of COSY and HMBC correlations were found to be in complete agreement with the proposed structure for $\mathbf{1}$ (Table 3). The name corticaglyceride was assigned to the new diglyceride (Tetracos-9-enoic acid 2-hydroxy-3octadec-9-enoyloxy-propyl ester).

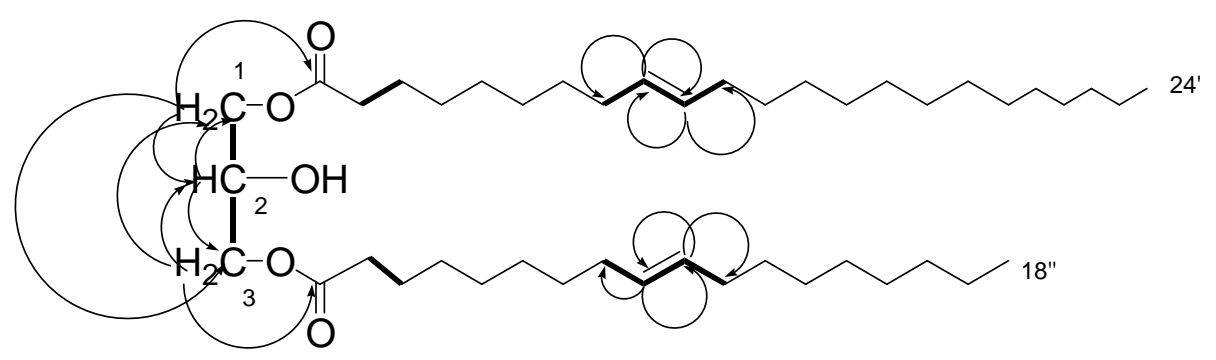

Fig. 1. Important HMBC (arrows) and COSY (bold) correlations of $\mathbf{1}$ 
Compound 2: The structure elucidation of $\boldsymbol{2}$ began with an analysis of its MS data that yielded an $\mathrm{m} / \mathrm{z}$ peak of 366 which combined with detailed analysis of the ${ }^{13} \mathrm{C}$ spectrum and DEPT indicated a molecular formula of $\mathrm{C}_{24} \mathrm{H}_{46} \mathrm{O}_{2}$ representing to two units of unsaturation. One of them was assigned as a carbonyl functional group for carboxylic acid where ${ }^{13} \mathrm{C}$-NMR of $\mathbf{2}$ showed a signal at $\delta 180.2$ (s). The other was suggested by appearance of signals at $\delta \quad 130.9$ and 128.8 assigned for olefinic carbons. This finding is consistent with the presence of a fatty acid. GLC analysis of the prepared methyl ester of compound $\mathbf{2}$ was carried out and by comparing the relative retention time of the peak with those of the pure available authentic standards. This compound is nervonic acid. Detailed analysis of COSY correlations were found to be in complete agreement with the proposed structure for nervonic acid (Table 4).

Compounds 3 and 4: These were identified as 24-methylene cholesterol and cholesterol by comparison of their spectroscopic properties with those reported in the literature. ${ }^{17 \& 18}$

Compound 5: The structure elucidation of $\mathbf{5}$ began with an analysis of its MS data. The high resolution ESI-TOF mass spectrum of 5 displayed a pseudo-molecular ion peak at $m / z 536.4807[\mathrm{M}+\mathrm{H}]^{+}$which combined with detailed analysis of the ${ }^{13} \mathrm{C}$ spectrum and DEPT indicated a molecular formula of $\mathrm{C}_{34} \mathrm{H}_{65} \mathrm{O}_{3} \mathrm{~N}$ representing three units of unsaturation. The presence of the fragment ions at $\mathrm{m} / \mathrm{z} 518.755$ and 500.4840 indicate loss of two $\mathrm{H}_{2} \mathrm{O}$ corresponding to two hydroxyl moieties in the molecule. The characteristic signals of 2-amino-1,3diol of the hydrocarbon chain were observed at $\delta 3.91(1 \mathrm{H}$, dt, $J=7.65$, 3.85), 3.70 (1H, br d), $3.95(1 \mathrm{H}, \mathrm{d}, J=$ $11.40)$, and $4.30(1 \mathrm{H}$, br $\mathrm{m})$ in the ${ }^{1} \mathrm{H}$ NMR spectrum and at $\delta 54.0(\mathrm{CHN})$, $62.5\left(\mathrm{CH}_{2} \mathrm{O}\right)$, and $76.8(\mathrm{CHOH})$ in the ${ }^{13} \mathrm{C}-\mathrm{NMR}$ spectrum, respectively. ${ }^{19}$ In addition, the ${ }^{1} \mathrm{H}$ NMR spectrum showed signals corresponding to aliphatic hydrocarbons at $\delta 0.88(6 \mathrm{H}, \mathrm{m}), 1.24$ (overlapped $38 \mathrm{H}, \mathrm{m}), 1.63(2 \mathrm{H}, \mathrm{m})$, $1.95(2 \mathrm{H}$, dd $J=10.30,6.85), 2.08$ $(2 \mathrm{H}, \mathrm{m}), 2.11(2 \mathrm{H}, \mathrm{dt}, J=21.10$, $6.50), 2.10,2.20(2 \mathrm{H}, \mathrm{t}, J=7.65)$ and four olefinic protons at $\delta 5.40(2 \mathrm{H}$, dt, $J=15.30,10.00 \mathrm{~Hz}), 5.50(1 \mathrm{H}, \mathrm{dd}$, $J=15.00,6.10 \mathrm{~Hz}), 5.79(1 \mathrm{H}, \mathrm{dt}, J=$ 15.00, $7.00 \mathrm{~Hz})$. The ${ }^{13} \mathrm{C}-\mathrm{NMR}$ spectrum showed signals due to two terminal methyl groups in aliphatic hydrocarbon chains at $\delta 14.2$, four olefinic carbons at $\delta 129.0,129.2$, 131.4, and 133.6 and an amide carbonyl at $\delta$ 174.0. Analysis of the ${ }^{1} \mathrm{H}-{ }^{1} \mathrm{H}$ COSY, HMQC and HMBC spectra led to the assignment of proton and carbon signals for $\mathbf{5}$. The position of the double bonds were confirmed by ${ }^{1} \mathrm{H}-{ }^{1} \mathrm{H}$ COSY spectrum between $\mathrm{H}-4 / \mathrm{H}-5, \mathrm{H}-5 / \mathrm{H}_{2}-6, \mathrm{H}_{2}-6 / \mathrm{H}_{2}-$ 7, $\mathrm{H}_{2}-7 / \mathrm{H}-8$ and $\mathrm{H}-8 / \mathrm{H}-9$ and also from $\mathrm{HMBC}$ of $\mathrm{H}-4 / \mathrm{C}-5\left({ }^{2} J_{\mathrm{CH}}\right), \mathrm{H}-$ $4 / \mathrm{C}-6\left({ }^{3} J_{\mathrm{CH}}\right), \mathrm{H}-5 / \mathrm{C}-6\left({ }^{2} J_{\mathrm{CH}}\right), \mathrm{H}-5 / \mathrm{C}-7$ 
$\left({ }^{3} J_{\mathrm{CH}}\right), \quad \mathrm{H}_{2} 6 / \mathrm{C}-8 \quad\left({ }^{3} J_{\mathrm{CH}}\right), \quad \mathrm{H}_{2}-7 / \mathrm{C}-8$ $\left({ }^{2} J_{\mathrm{CH}}\right), \quad \mathrm{H}_{2}-7 / \mathrm{C}-9 \quad\left({ }^{3} J_{\mathrm{CH}}\right), \quad$ H-8/C-9 $\left({ }^{2} J_{\mathrm{CH}}\right)$ leading to assignment of the C4/C-5/C-6/C-7/C-8/C-9 (Fig. 2). The position and geometry of the double bonds were confirmed by ${ }^{1} \mathrm{H}-{ }^{1} \mathrm{H}$ COSY analysis and coupling constant data. The $J_{4,5}(15.00 \mathrm{~Hz})$ and $J_{8,9}$ $(15.30 \mathrm{~Hz})$ values indicating the trans geometry of the double bonds. Therefore, $\mathbf{5}$ was assigned as a new 4E, 8E-sphingadiene type ceramide, reported here for the first time. GCMS analysis of the fatty acid methyl ester of compound $\mathbf{5}$ was carried out after hydrolysis and yielded an eicosanoic acid methyl ester. These results suggested that $\mathbf{5}$ was an $\mathrm{N}$-acyl eicosanoic acid derivative of $\mathrm{C}_{14}$ amino alcohols. The chemical shifts of C-1 ( $\delta$ 62.5), C-2 ( $\delta$ 54.0), C-3 ( $\delta$ $76.8)$, and $C-1$ ' ( $\delta$ 174.0) were very similar to those of the neurotrophic ceramide (4E,6E,2S,3R)-2-N-eicosanoyl-4,6-tetradecasphingadienine, which was recently reported. ${ }^{19}$ This evidence indicated the absolute configurations at $\mathrm{C}-2$ and $\mathrm{C}-3$ to be $2 S$ and $3 R$, respectively. Accordingly, the structure of $\mathbf{5}$ was assigned to be (4E,8E,2S,3R)-2-N-eicosanoyl-4,8tetradecasphingadienine. Detailed analysis of COSY and HMBC correlations were found to be in complete agreement with the proposed structure for $\mathbf{5}$ (Table 5). The name corticaceramide was assigned to the new sphingolipid.

Biological evaluation of isolated metabolites revealed that compound $\mathbf{5}$ possesses "medium" anti-oxidant activity while compound $\mathbf{1}$ has "weak" anti-oxidant activity. Compound $\mathbf{3}$ was found to exhibit anti-inflammatory activity comparable to dexamethasone (Table 6).

The isolated lipids, sterols and fatty acids in this study are also expected to serve as biomarkers indicating the presence of biological activity as well as for chemotaxonomic purposes.

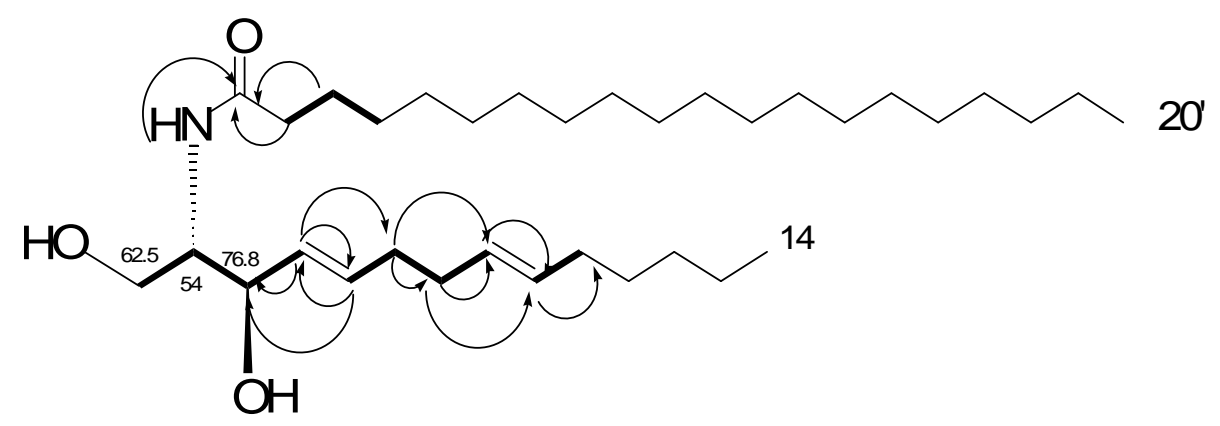

Fig. 2. Important HMBC (arrows) and COSY (bold) correlations of 


\section{Acknowledgment}

The authors wish to acknowledge the U.S.-Egypt Science and Technology joint fund, project number BIO8-002-013 for financial support. Thanks are also due to the Egyptian Environmental Affairs Agency (EEAA) for facilitating sample collection along the coasts of the Red Sea. The authors are grateful to Prof. Rob.W. M. van Soest, Faculty of Science, Zoological Museum, Amsterdam for taxonomic identification of the sponge.

\section{REFERENCES}

1- J. W. Blunt, B. R. Copp, M. H. G. Munro, P. T. Northcote and M. R. Prinsep, Nat. Prod. Rep., 20, 1 (2003).

2- D. J. Faulkner, M. D. Unson and C. A. Bewley, Pure and Appl. Chem., 66, 1983 (1994).

3- M. Kelly and T. Sammaai, Family Podospongiidae De Laubenfels. In Systema Porifera: A Guide to Classification of Sponges: N. A. Hooper, R. W. van Soest, M., Eds.; Kluwer Academic/Plenum: New York, Vol. 1, 2002, pp 698-699.

4- I. Spector, N. R. Shochet, Y. Kashman and A. Groweiss, Science, 219, 493 (1983).

5- T. R Hoye, S. E. N. Ayyad, B. M. Eklov, W. T. Hashish Shier, K. A. El Sayed and M. T. Hamann, J. Am. Chem. Soc., 124, 7405 (2002).

6- B. Vilozny, T. Amagata, S. L. Mooberry and P. Crews, J. Nat. Prod., 67, 1055 (2004).
7- S. Ahmed, S. Khalifa, M. Mesbah, D. Youssef and M. Hamann, 26 ${ }^{\text {th }}$ African Health Sciences Congress, Abs., 183 (2005).

8- K. A. El Sayed, D. T. A. Youssef and D. Marchrtti, J. Nat. Prod., 69, 219 (2006).

9- C. C. Parrish, T. A. Abrajano, S. M. Budge, R. J. Helleur, E. D. Hudson, K. Pulchan and C. Ramos, In: The Handbook of Environmental Chemistry (P. Wangersky eds.) SpringerVerlag Berlin Heidelberg Press, Vol. 5, Ch. 8 (2000).

10- G. Triola, G. Fabria`s, J. Casas, and A. Llebaria, J. Org. Chem., 68, 9924 (2003).

11- F. D. Gunstone, In, Fatty Acid and Lipid Chemistry (Chapman and Hall eds.) Chapman and Hall press, London, $1^{\text {st }}$ Edn., 1996, pp. 1-33.

12- P. Mason, The Pharmaceutical Journal, 273, 750 (2004).

13- British Pharmacopeaia, The Pharmaceutics Press, London (1973).

14- I. L. Finar, In: Organic Chemistry (Longman Groups Limited eds.) Longman Groups Limited Press, England $6{ }^{\text {th }}$ Edn., 1973, pp. 45-69.

15- Z. F. Mahmoud, N. A. Abd Elsalam and S. M. Khafagy, Fitoterapia, 51, 153 (1980).

16- S. Takamatsu, T. W. Hodges, I. Rajbhandari, W. H. Gerwick, M. T. Hamann and D. G. Nagle, J. Nat. Prod., 66, 605 (2003). 
17- T. S. Rao, B. Sarvani, D. V. Rao and C. B. Rao, Indian J. Chem., 35B, 1356 (1996).

18- H. A. Kalinowski, S. Berger and S. Braum, ${ }^{13} \mathrm{C}-\mathrm{NMR}$ Spektroskopie (George Thieme Verlag ed.) Stuttgart, New York, Vol. 2, 1984, p. 393.
19- H. C. Kwon, K. C. Lee, O. R. Cho, I. Y. Jung, S. Y. Cho, S. Y. Kim and K. R. Lee, J. Nat. Prod., 66, 466 (2003). 